\title{
SUCCESS AND FAILURES OF CROSSING FACILITIES FOR PEDESTRIANS
}

\author{
Aishwarya Fadnavis ${ }^{1}$ \\ ${ }^{1}$ Architect, Architecture, Bharati Vidyapeeth College, Maharashtra, India
}

\begin{abstract}
Walking is the most ancient mode of transport. But in today's world, there is a tendency to underestimate the pedestrian population. This article emphasis on the study of pedestrian crossing facilities such as underpasses \& overpasses and aspects involved in these facilities. This also helps to understand the need of underpasses and overpasses considering traffic movement of that area; purpose and functionality of underpasses and overpasses depending on the users. Population around India frequently shows the use of pedestrian crossing facilities in many areas with heavy flow of traffic.
\end{abstract}

Key Words: Pedestrians, underpass, design, and conditions

\section{INTRODUCTION}

In order to study the pedestrian crossing facilities, study of a particular city is done. It includes the issues involved in the usage of these facilities and solutions for the same. This will directly help the city to be pedestrian friendly and enhance the experience on road. Pune city is found evolving gradually into a city of traffic disorder and congestion due to increase in number of two wheelers and four wheelers on the road. Along with the increasing volume of traffic in Pune rises the struggle between different participants who contribute to the traffic congestion. This congestion varies from time to time. Pedestrians are most susceptible users in this traffic congestion who needs sustenance. The Pune city is expected to be for people but now has become city for vehicles. In the arena of pedestrian essentials, there are less facts and lack of awareness based on methodical documentation, observations and analysis. Such methodical documentation, observations and analysis will brace the role of pedestrians in the process by the governing bodies which requires verdict making for their (pedestrian's) assistance. Also Pune Municipal Corporation should undertake such studies before proposing more pedestrian facilities such as overpasses and underpasses, walkways, heritage walkways.

- Aim- To analyze and understand pedestrian insight about the crossing facilities i.e. underpasses and overpasses. The purpose of this analysis is to find out the reasons for the success and failures of these facilities along with the solutions to these issues.

- Objective- To study overpasses and underpasses are ideally constructed for safety, convenience, due to lesser number of steps, their virtuous condition, decent lighting to ensure safety, maintenance and preservation, security and more rapid movement which saves time of the pedestrians.

- Hypothesis- The reason for studying, documenting and analyzing this facilities is that, they are said to be dedicated to the security or protection and time saving factor for pedestrians are highly expensive as a construction and inconvenient to use. Along with this, there are certain other factors such as maintenance, social safety, visibility, time saving, design etc. which adds to the concern for the use of these facilities. There are scarce circumstances in which such structure are indeed mandatory and receiving pedestrians securely through the crossing facility is not a worthy sufficient reason for the determination of the crossing facilities.

\section{LITERATURE REVIEW}

Parisar, an organization in Pune carried out a study with an aim of getting a response from the pedestrians who regularly use facilities such as over bridges, under bridges and crossovers. The survey includes pedestrians using these underpasses and overpasses and those crossing at grade. The preferences of pedestrians was zebra crossing in most of the areas considering safety, convenience and most important time saving. Underpasses and overpasses are ideally constructed for safety and convenience of the pedestrian.

Most people opted for foot underpass as the facilities were mostly safe and convenient for all age groups, owing to lesser number of steps, good condition, good natural lighting, safety and faster movement.

Overpasses are also located in different areas in Pune such as Paud road, Satara road. Pedestrians find overpasses difficult to use for all age groups because of the huge number of stairs. As in case of the overpass near S.N.D.T college is viewed as being inconvenient for elders and children, the main reason being the number of steps ( 84 steps) required to climb, which is tiring and time consuming. The crossing locations should provide for safe and comfortable movement. Pedestrian crossings are provided along the minor intersections, mid blocks to provide safe crossings for the pedestrians. Important aspects to be considered in the provision of pedestrian crossing facilities like underpasses and overpasses are as follows

- Crossings should be at right angles to the carriageway. 
- Crossings should be illuminated with proper visibility.

- Crossings should be place intelligently along the pedestrian desire lines.

- Provided with other accessories like warning signs, markings, information signs etc.

- Road safety should be done before opening the road for traffic.

The concept is to give priority to the movement of pedestrians when he/she steps on a zebra crossing, but with cities like Bangalore, Delhi, and Pune which are ranking high in traffic violations in the country, the priority is always with the motor vehicles. The most vital point in considering the pedestrian's crossings and the nature of traffic on road is the traffic flow speeds. Major roads have peak hours and the speed profile is not constant. Urban roads in India due to high congestion and the above reasons a suitable crossing facility for the pedestrians is a necessity for their safety.

The paper titled "Challenges Faced in Planning, Design \& Construction of Grade Separator near Apsara Border, Delhi", emphasizes on the use of underpasses and overpasses in Delhi. They take into account the different age groups who use the facilities by providing escalators and lifts along with staircase. The underpasses are developed and maintained by the authorities in the form of shopping centers such that people are encouraged to use the stairs. One of the most important factors which affect the functionality of these underpasses and overpasses is its integration with metro stations and petrol pumps.

There are various factors specifying use of underpasses and overpasses in Pune-

2.1. Traffic volume, traffic bulk on Karve road increases as the time duration changes, during my observation. Initially there was a possibility of crossing the road safely but later it got very substantial- hence crossing for the pedestrians became challenging and people had no option but to use the overpass.

2.2. Design of the underpass and overpass, if the overpass and underpass are intended, planned and designed according to the needs of its users, it gets generally acknowledged and attains success for not only the pedestrians but also the vehicles on the road.

2.3. Location of the facility, the pedestrians walking on the footpath or the walkway come routinely across the entry of the underpass or overpass. The signages on the road also help the pedestrians to locate the facility.

2.4. Maintenance of the facility, the underpass and overpass are preferred by the users only if they are clean and secure.

2.5. Visibility of the underpass and overpass, the entry must be effortlessly distinguishable. This is predominantly accurate for underpasses as they are not at grade. The pedestrian facility is hard to interpret on the road and no signages does not save time for the pedestrians, even the local residents cannot articulate existence or disbelief the approachability.

When it comes to the preferences of pedestrians among three kinds of facilities, it can be observed that zebra crossing is the most pursued and first choice of pedestrians, while the overpass has minimum favored possibility. From the studies conducted, reasons for using underpasses are, the facilities were largely found to be secure and appropriate, due to less number of steps, their virtuous condition, decent lighting, security and quicker movement or time saving. Along with this, as in case of overpasses, the purpose of security from traffic was served by these facilities but this was at the cost of accessibility, closeness and interval.

Several researchers have studied pedestrian behavior at crossings. Hamed (2000) studied the factors influence a pedestrian's waiting time and frequency of attempts to cross the streets. He found that the pedestrian's expected waiting time has profound influence on the number of attempts needed to successfully cross the street. As stated in "Proceedings of the Eastern Asia Society for Transportation Studies, Vol.7, 2009", the pedestrian underpass is near to the bus stand and AIIMS hospital in Delhi. This pedestrian underpass had many medical and other shops. Some people went into the underpass to buy medicines or make other purchases. This happened on the AIIMS side since the hospital connects to the underpass and since many shops are near its exit. This survey also shows that $22 \%$ of pedestrians accepted a risk despite the presence of a nearby pedestrian underpass. This survey also showed an aspect of pedestrian behavior in the distance they are willing to use an underpass. The need for an underpass and overpass was felt for more than two decades. Long queues at the intersection, frequent jams with traffic struck for hours with pedestrians risking their lives to cross the road, were common sight at the intersection.

In an analysis conducted by an organization 'Parisar' in Pune, stated the observation of how pedestrian distinguishes the transformation in the traffic condition in Pune over the last few years where none of the pedestrians felt that the circumstances about the traffic has gone better. Pedestrians gave sentence in Pune Today saying that they do not feel recognized at all, neither on the road nor in the plan development by the authorities adding that they have to run like mice on the roads to cross it to avoid getting in an accident and traffic disturbance. Which is completely true especially in case of Pune because Pune has one of the worst traffic in India considering the destructive cocktail of high accident, vehicular pollution, traffic violations and motorization levels. In a city like this it is very necessary to consider the pedestrian crossing facilities or else the pedestrians would have to shift to other mode of transport.

\section{METHOD}

As a first step, an area was selected which has heavy traffic during peak hours, more pedestrians due to more number of bus stops and more colleges in neighborhood. A $9 \mathrm{~km}$ stretch of road from Nal stop to Chandani Chowk, Pune was taken 
for study. A list of all public underpasses and overpasses was created. The focus of the analysis was on pedestrian's perception and pedestrian's road safety along with success and failures of the same. Field visit, sections along the road, photographic documentation and interviews were undertaken thereafter.

3.1. Field Visit- Each pedestrian facility or underpass and overpasses were located on the base map along with neighborhood for reference. Each of these underpass and overpass was visited and various factors such as security, maintenance and general were studied there along with this the overall traffic of that area with the sections were also studied.

3.2. Drawings and Sections- These helped along with the field visit to analyze the concerns of the pedestrians in that area or facility.

3.3. Photographic_Documentation- Photography was done to identify the problem. The photographic documentation was done at different duration of time i.e. morning, evening and during the peak hours of traffic.

3.4. Interviews- This procedure included the response from pedestrians who frequently use these underpass and overpass along with other facilities for especially security, accessibility along with the design, lighting and maintenance. To appreciate the requirements of pedestrians and also to know what they think about these underpasses and overpasses along with other facilities as an entire. For all these numerous purposes and achieve these goals an interview form was created to get all mandatory statistics from the pedestrians or the users of these facilities. A total of 24 pedestrians using these underpasses and overpasses were interviewed in the process across all these underpasses and overpasses.

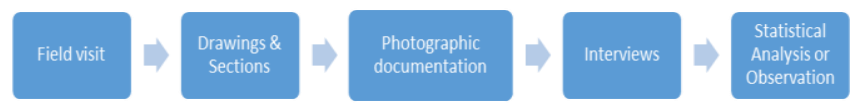

\section{PRIMARY DATA}

Various reports studying these facilities and their conditions emphasizes on the functionality of the pedestrian crossing facilities. They suggested that a healthy functionality pedestrian arrangement or structure permits the pedestrians to be safe while crossing the road even during peak hours and busy hours on the road and it would be a strong commitment towards an individual's approachable traffic strategy made by the local authorities. It can be understood from the studies and analysis that-

4.1. The first factor is the design of the pedestrian facility, if an underpass or overpass is designed considering the needs of its users, it gets used by the pedestrians during peak hours of the day. Though the overpass near S.N.D.T College, Nal stop is used on a large scale but is inconvenient due to its design of steps.
4.2. The second factor is the location, the underpass near Kinara hotel is an example of a good case i.e. the pedestrians walking on the walkway come routinely in front of the entry of the underpass. On the contrary, the overpass near S.N.D.T College is an example of critically located pedestrian assembly and a facility is used much by the pedestrians. People on Karve road who want to pass Gulmohor Path have first to turn onto Gulmohor Path and climb about 88 steps to cross a two-lane road which has traffic congestion due to various modes of transport like the buses, four wheelers, trucks, and two wheelers etc.

4.3. The preservation of the facility, it is an important factor being responsible for a pedestrian facility to be in use or not by the pedestrians. Characteristics that determine the success of these facilities include how the natural light is and radiance. Along with this also how cleanly and regularly it is maintained, sustained and retained after changes in the design along with the security within the underpass and overpass.

4.4. The distinguishability of the underpass and overpass, the entry must be effortlessly distinguishable and discreet. This is particularly important factor for underpasses since they are underground.

It is suggested that the local authorities must contact the local masses using these facilities before they adopt to announce these facilities on the road. This could be done by coordinating with civil society organizations and other organizations. This surges dynamic contribution and involvement of residents in the development eventually contributing to the progress and advancement of their city. Latest techniques are used for construction of these bridges for future. Karve road is overcrowded with vehicles, pedestrians don't have option to cross the road safely.

The S.N.D.T college overpass is often used, it is convenient and safest way to cross the road. It is mostly used at the college's beginning and end timings i.e. 9:30a.m and 5:00p.m peak hours after 5p.m till 9p.m as the road traffic increases also due to heavy vehicles, in this timing most people use this facility. Near this overpass, there are five bus stops hence crowded by the pedestrians. Along with all these an auto stand facility in also found for the pedestrians and especially for women, a toilet facility is given in this overpass.

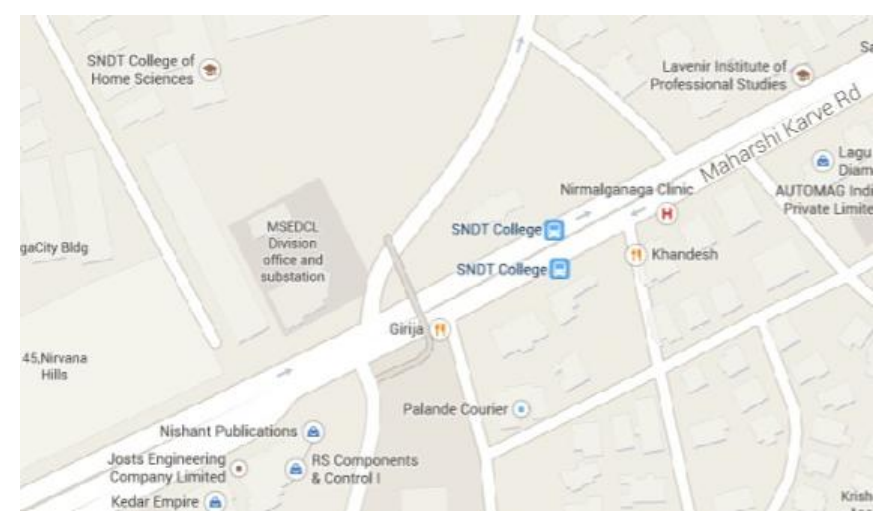

Location of the overpass near S.N.D.T College, Kothrud, Pune 
The underpass near Kinara Hotel is mostly used by the visitors of Sahyadri hospital and Vanaz Company. Also a noticeable auto rickshaw stand is seen near the underpass which increases its functionality.

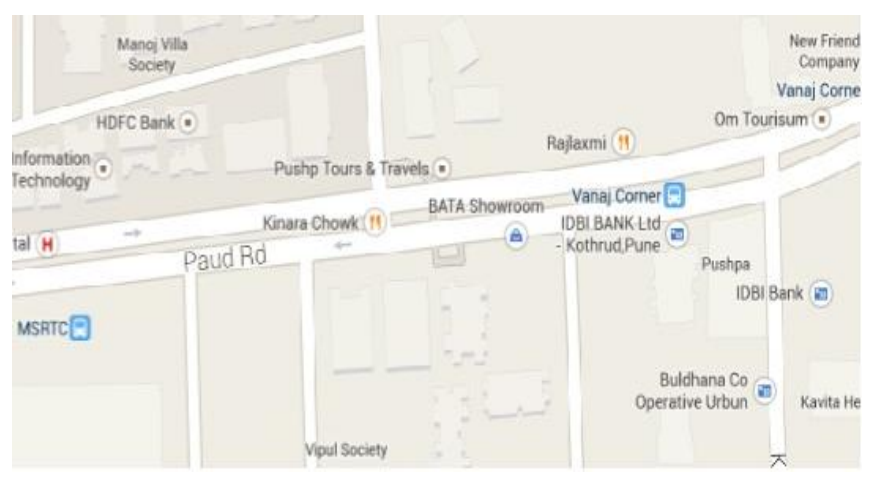

Location of the underpass near the Kinara hotel, Kothrud, Pune

\section{PROBLEM IDENTIFICATION}

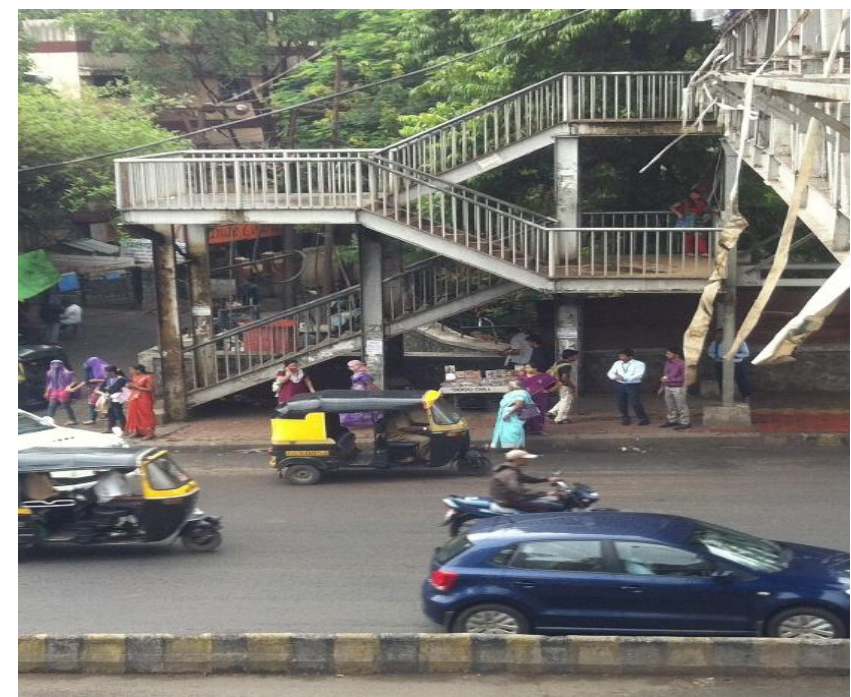

This image shows the traffic near the college and the overpass during the peak hours and the other picture shows the use during this timing.

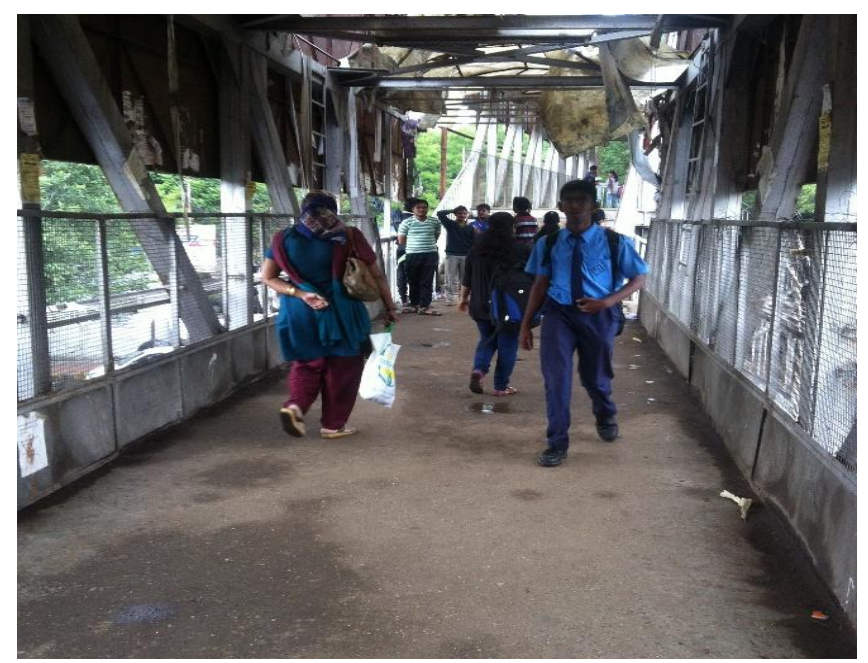

Use of S.N.D.T overpass during peak hours and used by college students, pedestrians etc

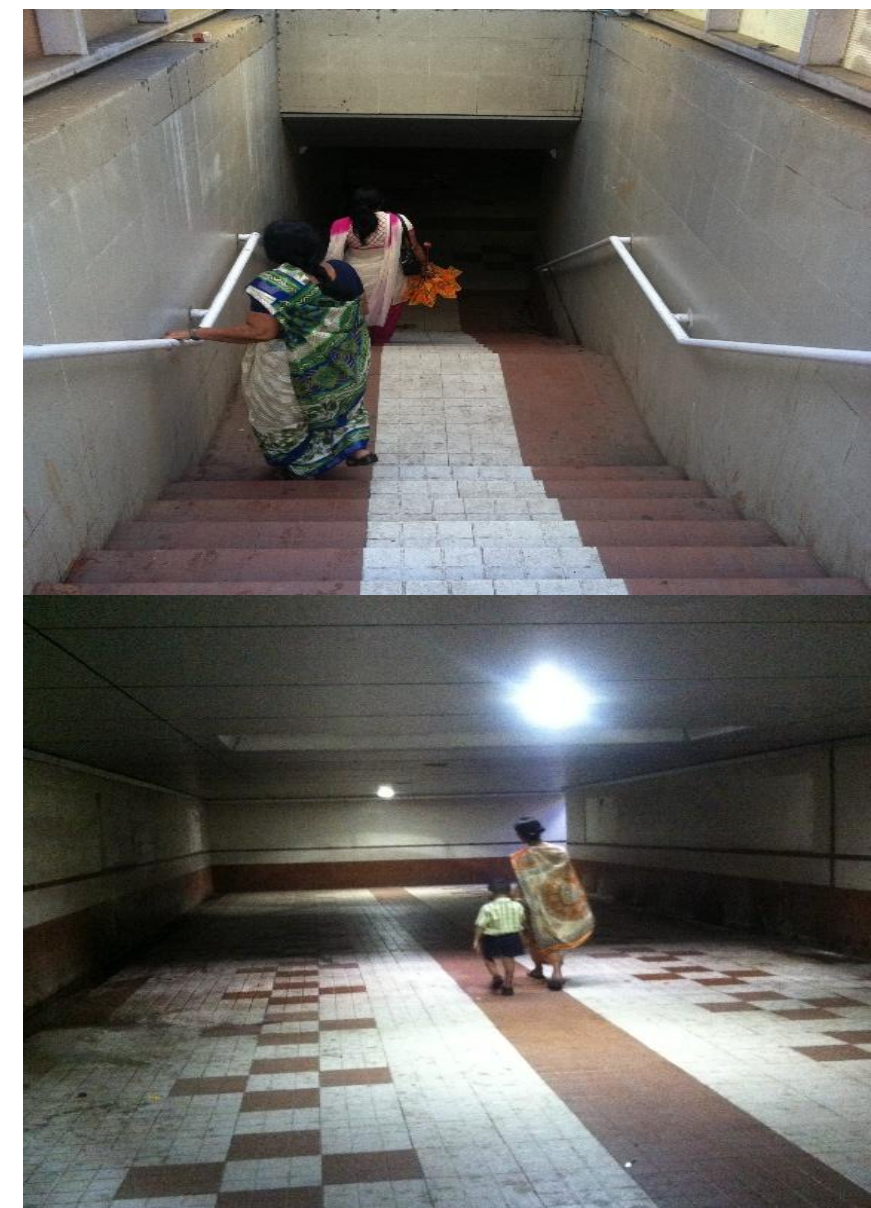

The above picture shows less number of people using the underpass due to water collection in monsoon and less natural light and no illumination during evening/night.

Detail plan of the underpass near the Kinara hotel which is found convenient in case of design and location but lacks the facilities, maintenance and performance in monsoon. The underpass is near the Kinara Hotel and is $6 \mathrm{~m}$ wide. The underpass is located near the Sahyadri Hospital and Kinara Hotel. Generally very crowded and mostly used by the people coming to hospital nearby and Vanaz company, but still is found to be unsafe socially for women especially during evening or night hours. It infers that an underpass or overpass is mostly used when the location is suitable for the facility. The crossing facility is proposed or developed considering the location. This underpass lies between the Vanaz Company and a hospital which makes the space more usable and dynamic in function. The most important factor being the light and ventilation inside an underpass is a major issue which results in unsafe conditions in the space.

Overpass is located near S.N.D.T College which is quite inconvenient due to high number of steps and low maintenance. Performance of this overpass weakens in monsoon due to poor ceiling condition. Though it is used mostly in peak hours of traffic. It continues to be inconvenient for elders and children to use this facility. As considerable light during day and evening is present, it is observed that this facility is used more than the underpasses nearby. Social safety for women is seen in this overpass. 


\section{STATISTICAL \\ OBSERVATIONS}

ANLYSIS

AND

6.1. Condition of ceiling, when the users were asked about the condition of the ceiling especially for overpasses, $58 \%$ people said that it is average and $34 \%$ said it is good while only $8 \%$ said it can improve.

\section{CONDITION OF CEILING}

average good acan improve

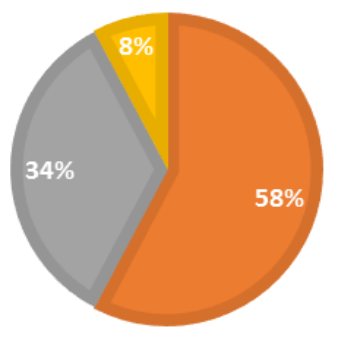

Observation- the condition of ceiling and the materials used for the roof should be changed as in case of overpass, the overpass is not protected from the rain and hence the water starts collecting making the floor slippery. It is observed that the overpass which is not hygienic and fresh is not chosen by the pedestrian, but this facility becomes mandatory to use by the local people because crossing on the road during the peak hours is not possible and causes traffic congestion at grade.

6.2. When asked whether the underpass or overpass is time saving or not, $83 \%$ users said Yes saying that the underpass and overpass does save their time but $17 \%$ users said that they have to take a longer route to reach the facility.

\section{CONDITION OF CEILING}

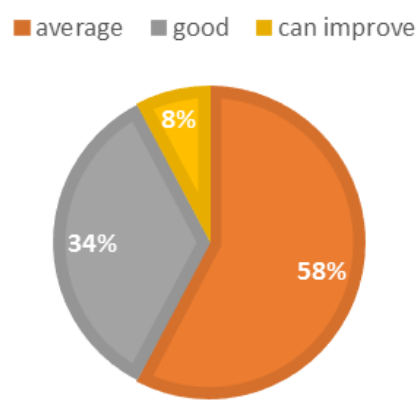

Observation- the condition of ceiling and the materials used for the roof should be changed as in case of overpass, the overpass is not protected from the rain and hence the water starts collecting making the floor slippery

6.3. When asked whether the underpass or overpass is time saving or not, $83 \%$ users said Yes saying that the underpass and overpass does save their time but $17 \%$ users said that they have to take a longer route to reach the facility.

\section{TIME SAVING}

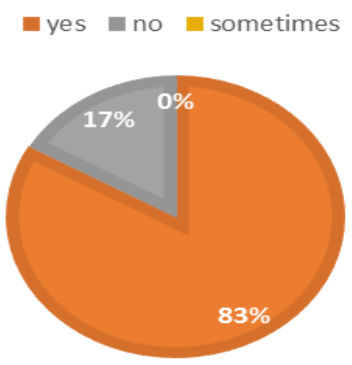

Observation- it was observed and studied that these facilities are not on the direct way but they have to cross the street and it does not save time.

6.4. One of the major concerns in underpass and overpass is natural light and illumination, where 54\% pedestrians said that it is average and $25 \%$ said it is good while $21 \%$ said it can be improved and should be improved.

\section{NATURAL LIGHT/ILLUMINATION}

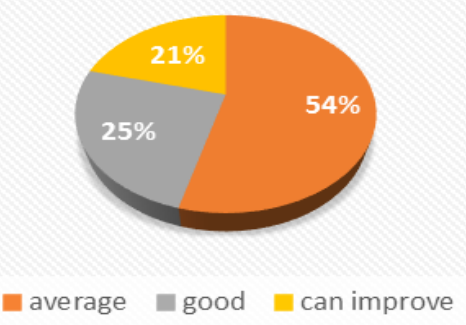

Observation- it was observed that due to the location and design, there is lack of natural sunlight in the underpass which causes visibility issues to old people and children. Women face the lack of safety when there is less illumination and thus creating uncomfortable atmosphere.

6.5. This factor was especially for women, where only $30 \%$ users found it safe for women while $70 \%$ users said it is not safe socially for women especially underpasses.

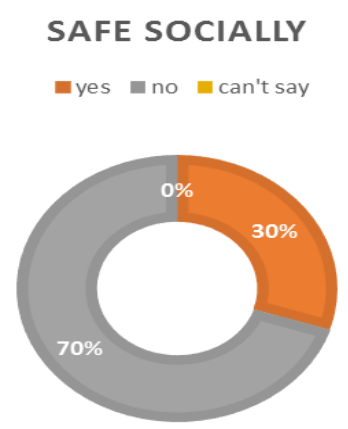

Observation- as pedestrians needs protection on the road during traffic chaos. Constant, unbroken and unhindered footpaths, on every street are unavoidability, as also well planned and considered according to the needs and executed pedestrian trips. 
6.6. When the pedestrians using the facilities were asked about the locations of these facilities, about $55 \%$ users said it is difficult to locate the underpass or overpass in an area. Just $10 \%$ users said it is easy to locate while $35 \%$ added that they want a change and the signs should improve.

\section{LOCATION OF FACILITY}

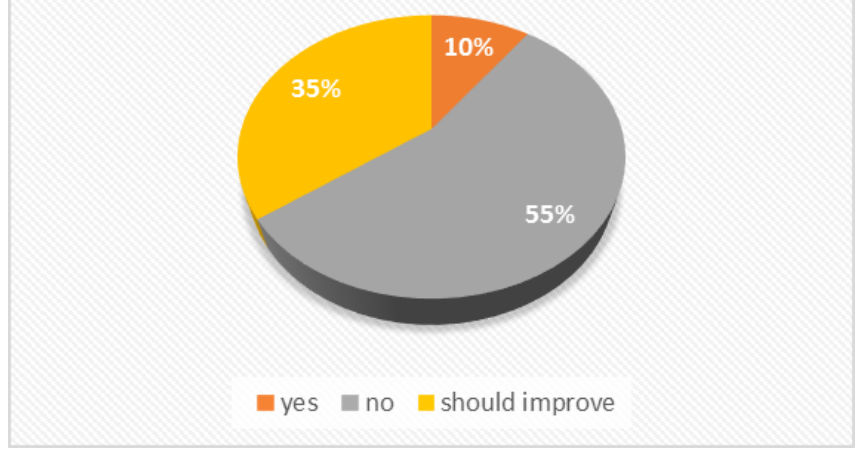

Observation- as a accessibility part, people are likely to choose the informal way, so that walking is made easy for them. That includes walkable footpaths, sheltered walkways by streets or street furniture, sufficient space to permit other pedestrians when there is pedestrian chaos also on the walkways. This is especially found near the bus stops and rickshaw stands, pavement edges should not be broken and angular (preferred to be rounded), benches for recreation and most imperative of all the dodging of steps.

\section{SUGGESTIONS}

New concepts being introduced in the cities, like pedestrian signals, must be introduced along with explanation and supervision to the citizens- both motorists and pedestrianswho are expected to be furthered from it.

By setting up a pedestrian Policy, Corporation would send an indication that they fairly try to transform the current city into a much civilized or habitable city. This policy for the pedestrians should meet the following criteria:

7.1. Direct Pedestrian Routes- the shorter the way, the more people are likely to stay pedestrian and use the crossing facilities which will eventually help in future to reduce the traffic congestion on the roads by the vehicles.

7.2. Good Connectivity- e.g. public transport close to the housing area, hospitals, colleges so that walking and using these facilities becomes the main passage and underpasses/overpasses near this bus stop will surely advantage the pedestrians and assist them in saving the time. These facilities must be located considering the whereabouts and preferably should connect significant spaces together on either sides.

7.3. Convenience- people are likely to choose the informal way, so walking should be made convenient. Condition of the pedestrian facilities should also be considered which includes potholes, manholes, trees etc. on the walkways. The parking of two wheelers on the walkways should be avoided by increasing the height of the walkways from the road level. Also the walking facilities should be enhanced by adding street furniture, outdoor sitting area, and food joints. A market place within or along these facilities will summon pedestrians to use the facility.

7.4. Safety- the pedestrians need the most and best security. Uninterrupted and unhindered footpaths along the busy streets is mandatory which includes free from potholes, manholes, etc. These footpaths should lead to the crossing facilities like the underpasses and overpasses with proper pedestrian signals and signage. Interpretation of these facilities is equally important for the smooth functioning of the space.

7.5. Design of these facilities- The pedestrian crossing facilities should be well designed and implemented. The design must be barrier free i.e. effortlessly accessible by the older people and the people with wheel chairs. This design also includes suitable number of steps especially for an overpass where the height should be restricted to a certain limit. Roofing material should be chosen as per design and it should be made sure that the overpass remains working during monsoon. Similarly, underpass should have proper drainage line so that the water does not remain logged inside the space making it unusable.

\section{CONCLUSIONS}

For a city where the chief inhabitants uses community transport and walks throughout the various areas within the city, the local authorities are more involved in high costs and time consuming projects for the motorists. It is said that by constructing underpasses for pedestrians trip through the busy roads is a deteriorating interchange. Pedestrians have a superior assertion to roads as vehicles but it should be understood that in the future the chaos of the four wheelers and public transport system is going to increase which needs a solution from now. Encouragement among the people is necessary in every developing city in India. The affinity to impulse pedestrians into underpass or overpass and permit vehicles to use the road is a deteriorating interchange. It means, it is very disputed and doubtful to pressurize people to devote supplementary exertion climbing stairs in case of overpass and get down the stairs in case of underpass to get to the other side of the road which maybe two lane or even six lane. Even for roads like Karve Road there are keys for harmless pedestrians crossing at grade.

An overall absence of responsiveness among residents about their privileges as a pedestrian was studied and concluded. The pedestrians and local residents must be vigorously participating in groups and must answer the local authorities and the involved organizations in these issues via newspapers, e-mails, social network and social media etc. to request what they want from theses governing and nongoverning bodies. The authorities must refer the people and their needs before they choose to announce not only the pedestrian facilities but also anything new in the city regarding the betterment and progress of the city 


\section{REFERENCES}

[1]. Times of India, June $10^{\text {th }}, \quad 2010$ : http://timesofindia.indiatimes.com/india/Reddysuggests-congestion-tax-to-cure-carmania/articleshow/6029940.cms

[2]. Guidelines for Pedestrian Facilities (1989), Indian Road Congress- 103-1988

[3]. Das, S., Manaski, C., Manuszak, M. (2003). Walk or Wait? An Empirical Analysis of Street Crossing Decisions. Discussion Paper in Economics. Indian Statistical Institute (ISI 2003)

[4]. Hamed, M. M. (2000). Analysis of Pedestrians' Behaviors at Pedestrian Crossings. Safety Science, 38, 63-82.

[5]. Kaplan, E. L. And Meire, P. (1958). Nonparametric Estimation from Incomplete Observations. Journal of the American Statistical Association, 53, 457-481.

[6]. Cox.D. R.(1972), "Regression Model and Life Tables", Journal of the Royal Statistical Society, Series B, 34, $187-220$.

[7]. Al Madani.H, Al Janahi,A,(2005), Personal Exposure Risk Factors in Pedestrian Accidents in Bahrain, Safety Science

[8]. Akbar and Sudhir,(2007), Dissecting Bangalore Traffic, World Transport Policy and Practice, Vol 13.2

[9]. Hermann Knoflacher,(2007), "Success and Failures in Urban Transport Planning in Europe- understanding the transport system", Sadhana Vol. 32, Part 4

[10]. Shankar.V.N, Ulfarsson.G.F, Pendyala.R.M, and Nebergall.M.B,(2002), "Modeling Crashes involving Pedestrians and Motorized Traffic", Safety Science, 41,627-640

\section{BIOGRAPHIES}

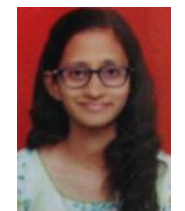

Aishwarya Fadnavis

I am a student of architecture from Pune. Analyzing the issues and suggesting solutions is my field of interest supported by statistics and my observations 